\title{
SECABA-RANK, HERRAMIENTA ONLINE PARA ANALIZAR Y EVALUAR BIBLIOTECAS
}

\section{Secaba-Rank: An online tool to analyze and evaluate libraries}

\author{
Pedro Lázaro-Rodríguez, Javier López-Gijón, Sergio Alonso, María-Ángeles \\ Martínez-Sánchez y Enrique Herrera-Viedma
}
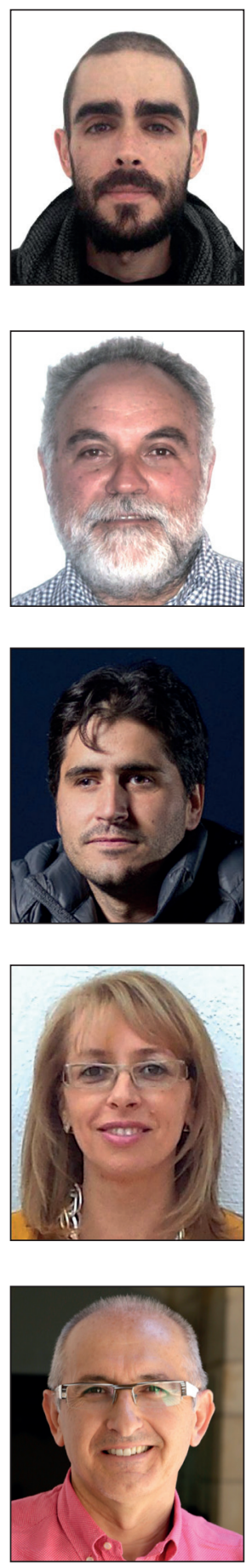

Pedro Lázaro-Rodríguez es licenciado en Documentación y máster en Información y Comunicación Científica por la Universidad de Granada (UGR). Está realizando su tesis doctoral sobre bibliotecas públicas y universitarias españolas contratado por la UGR dentro del programa de Formación de Profesorado Universitario (FPU) del Ministerio de Educación, Cultura y Deporte de España. Es miembro del laboratorio de investigación Secaba-Lab.

https://orcid.org/0000-0002-8756-0507

Universidad de Granada, Departamento de Información y Comunicación Campus Cartuja, Edificio Máximo. 18071 Granada, España pedrolr@ugr.es

Javier López-Gijón es doctor en Documentación por la Universidad de Granada (UGR) y profesor del Departamento de Información y Comunicación de la misma. Es miembro del laboratorio de investigación Secaba-Lab, donde ha sido investigador principal de varios proyectos y coordinador de estudios de evaluación de calidad de bibliotecas.

https://orcid.org/0000-0001-6517-8991

Universidad de Granada, Departamento de Información y Comunicación Campus Cartuja, Edificio Máximo. 18071 Granada, España jgijon@ugr.es

Sergio Alonso es doctor en Ciencias de la Computación por la Universidad de Granada (UGR) y profesor titular del Departamento de Lenguajes y Sistemas Informáticos. Su investigación e intereses van desde las bibliotecas digitales, públicas y universitarias, hasta la bibliometría, sistemas basados en lógica difusa y recomendación para la ayuda en la toma de decisiones.

https://orcid.org/0000-0001-6620-328X

Universidad de Granada, Departamento de Lenguajes y Sistemas Informáticos Periodista Daniel Saucedo Aranda, s/n. 18071 Granada, España zerjioi@ugr.es

María-Ángeles Martínez-Sánchez es doctora en Ciencias Sociales, máster en Información y Comunicación Científica y diplomada en Trabajo Social por la Universidad de Granada (UGR). Es profesora del Departamento de Trabajo Social y Servicios Sociales y su investigación más reciente gira en torno al análisis y estudios bibliométricos en la disciplina del trabajo social.

https://orcid.org/0000-0001-7523-3827

Universidad de Granada, Departamento de Trabajo Social y Servicios Sociales San Jerónimo. 18001 Granada, España mundodesilencio@ugr.es

Enrique Herrera-Viedma es profesor de Ciencias de la Computación e Inteligencia Artificial en la Universidad de Granada y Vicerrector de Investigación y Transferencia. Sus líneas de investigación relacionadas con la Biblioteconomía y Documentación se centran en recuperación de información, bibliometría, bibliotecas digitales, evaluación de webs, sistemas de recomendación y medios sociales. Es miembro del comité ejecutivo de la IEEE SMC Society y editor asocidado de las revistas IEEE Transactions on systems, man, and cybernetics: Systems, Knowledge based systems, Soft computing, Fuzzy optimization and decision making, Applied soft computing, Journal of intelligent y Fuzzy systems, and information sciences. Su índice h es 57 (WoS) y está presente en los listados de investigadores altamente citados de Clarivate Analytics.

https://orcid.org/0000-0002-7922-4984

Universidad de Granada, Departamento de Ciencias de la Computación e Inteligencia Artificial Periodista Daniel Saucedo Aranda, s/n. 18071 Granada, España viedma@decsai.ugr.es 


\title{
Resumen
}

Secaba-Rank es una herramienta digital abierta que incluye Secaba-Rank Universitarias y Secaba-Rank Públicas con el objeto de conocer y evaluar la realidad de las bibliotecas universitarias y las redes de lectura pública españolas para su mejora. La metodología empleada basada en la teoría general de sistemas ha permitido el desarrollo de indicadores de segundo nivel centrados en la eficiencia, facilitando el benchmarking y la localización de los modelos de buenas prácticas para la mejora constante y especialmente de las que más lo necesitan. Secaba-Rank se convierte así en un punto al que acudir para obtener información por parte de interesados y gestores para la toma de decisiones y punto del que partir en futuras investigaciones. Se presenta la herramienta y una muestra de los principales resultados.

\section{Palabras clave}

Bibliotecas universitarias; Bibliotecas públicas; Redes de lectura pública; Mapas de lectura; Eficiencia; Indicadores; Rankings; Indicadores de segundo nivel; Diseño de sistemas de información; Benchmarking; Secaba-Rank.

\begin{abstract}
Secaba-Rank is an online tool that includes Secaba-Rank Universitarias and Secaba-Rank Publicas, which can be used to evaluate both Spanish university libraries and public reading networks. Using a systemic approach, the methodology makes use of second level indicators focused on efficiency. This methodology facilitates the benchmarking of libraries to find models of good practice in order to improve. Secaba-Rank is an essential tool for decision making by researchers and managers in libraries.
\end{abstract}

\section{Keywords}

University libraries; Public libraries; Public reading networks; Efficiency; Indicators; Rankings; Second level indicators; Information systems design; Benchmarking; Secaba-Rank.

Lázaro-Rodríguez, Pedro; López-Gijón, Javier; Alonso, Sergio; Martínez-Sánchez, María-Ángeles; Herrera-Viedma, Enrique (2018). "Secaba-Rank, herramienta online para analizar y evaluar bibliotecas". El profesional de la información, v. 27, n. 2, pp. 278-288.

https://doi.org/10.3145/epi.2018.mar.06

\section{Justificación}

Se presenta Secaba-Rank, una herramienta digital abierta que incluye Secaba-Rank Universitarias y Secaba-Rank Públicas, con un doble cometido:

- dar a conocer los resultados de nuestro estudio sobre bibliotecas universitarias y las redes de lectura pública españolas tanto a interesados como a gestores;

- servir de base para futuras investigaciones al respecto.

http://secaba.ugr.es/rank

http://secaba.ugr.es/rank/universitarias

http://secaba.ugr.es/rank/publicas

El estudio se ha llevado a cabo desde una nueva metodología basada en la teoría general de sistemas (Von-Bertalanffy, 1969) concibiendo las bibliotecas y redes como sistemas, permitiendo el desarrollo de indicadores de segundo nivel para conocer su eficiencia y facilitando la localización de los modelos de buenas prácticas en un ejercicio de evaluación comparativa (benchmarking) para la mejora especialmente de las que más lo necesitan.

Tanto Secaba-Rank Universitarias como Secaba-Rank Públicas incluyen resultados para los años 2007 y los comprendidos entre 2010 y 2015 incluidos. Para el primer caso se han considerado las bibliotecas universitarias españolas con datos disponibles ofrecidos por la Red de Bibliotecas Universitarias Españolas (Rebiun) en su sección de estadísticas (Rebiun, s.f.). Para el segundo caso y entendiendo por redes de lectura pública el conjunto de bibliotecas públicas por provincias y/o comunidades autónomas, se han incluido las que disponen de datos ofrecidos por el Ministerio de Educación, Cultura y Deporte (MECD) en la página Bibliotecas públicas españolas en cifras (MECD, 2015).

\subsection{Antecedentes: evaluación, eficiencia y rankings en bibliotecas españolas}

La evaluación puede entenderse como una forma para conocer y mejorar la calidad de las bibliotecas (Gimeno-Perelló, 2008) y puede llevarse a cabo desde diversas perspectivas: subjetiva y objetiva (Fushimi, 2011). En el primer sentido y centrando el tema en España destacan estudios que utilizan la metodología Libqual+.

https://www.libqual.org/home

Su objetivo es conocer la calidad de bibliotecas según la opinión y satisfacción de los usuarios recopilando los datos por medio de encuestas (López-Gijón et al., 2010). Dentro de esta línea destaca el reciente desarrollo de sistemas de ayuda en la toma de decisiones basados en información lingüística difusa y aplicados sobre dicha metodología con el objetivo de mejorar la calidad de los servicios (Cabrerizo et al., 2015; 2017).

En el sentido de la perspectiva objetiva encontramos estudios que se centran en la evaluación de servicios de referencia (González-Fernández-Villavicencio; Cánovas-Álvarez; Arahal-Junco, 2014), la circulación de la colección (Rodríguez-Bravo; Rodríguez-Sedano, 2016), o la usabilidad de portales web (Muñoz-Egido; Vianello-Osti, 2017). En estos trabajos los datos se recogen de forma automatizada como mediciones de la realidad. También destacan trabajos en 
referencia al presupuesto de las bibliotecas analizando los posibles efectos de la crisis (Hernández-Sánchez; Arroyo-Vázquez, 2014; Simón-Martín; Arias-CoeIlo; Simón-Blas, 2016), y los que calculan el retorno de la inversión (ROI) para demostrar que las bibliotecas no son un gasto y sí más bien una inversión que produce unos beneficios netos (Taladriz-Mas, 2013).

En España se han planteado a lo largo del tiempo varios proyectos sobre evaluación de bibliotecas, sistemas y rankings muy concretos que cabe destacar. Algunos ejemplos en bibliotecas públicas fueron:

- Programa de Análisis de Bibliotecas $(P A B)$ de la Fundación
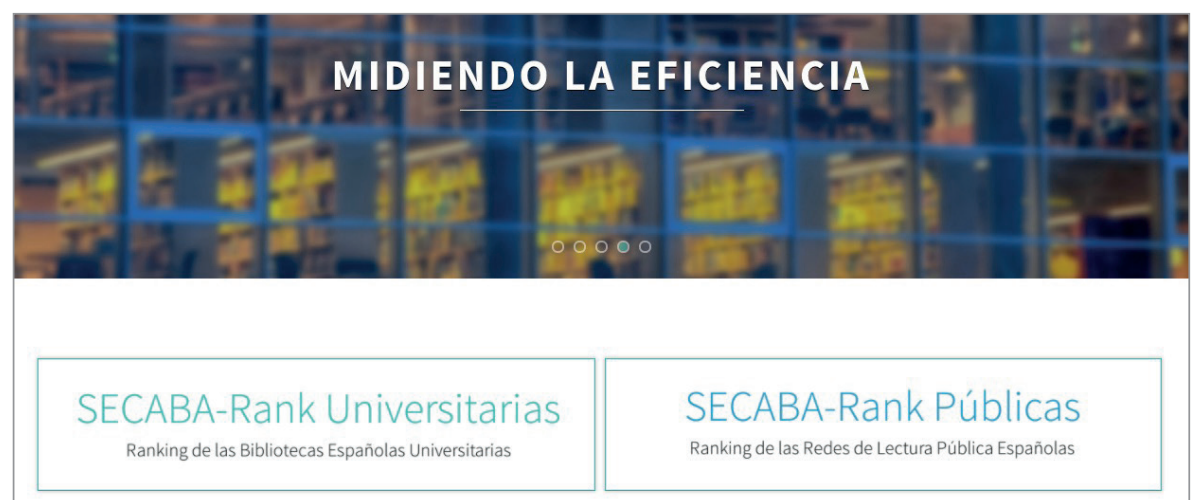
Bertelsmann, que facilitaba a

Figura 1. Secaba-Rank

http://secaba.ugr.es/rank las bibliotecas información en torno a unos 30 indicadores con el objetivo de su mejora poniéndolas en relación unas con otras y a sus directores (Motos, 2002);

- Proyecto Tibidabo del Consorci de Biblioteques de Barcelona $(C B B)$ y el Servei de Biblioteques de la Diputació de Barcelona, que surgió en 2003 con el objetivo principal de la mejora mediante la comparación y la cooperación entre las mismas ( $C B B$, s.f.).

Tal y como se puede comprobar en las fuentes mencionadas, el $P A B$ expiró en 2000 y el Proyecto Tibidabo ya no está operativo, pudiéndose consultar informes desde 2000 a 2009.

En cuanto a bibliotecas universitarias cabe destacar, por ejemplo, el ranking incluido en el Atlas digital de la España universitaria con indicadores sobre infraestructuras, recursos bibliográficos y usuarios para detectar las fortalezas y debilidades de las bibliotecas universitarias y científicas españolas (Reques-Velasco, 2006).

También y más actual se puede mencionar en un contexto más internacional un método creado para evaluar las características estructurales y dinámicas de las bibliotecas universitarias en el espacio digital (Martínez-Cardama; Caridad-Sebastián, 2016).

Algo que llama la atención desde la revisión de estos proyectos, métodos y rankings es que la mayoría atienden tan sólo a una parte o servicio de las bibliotecas y son pocos los que relacionan sus diferentes dimensiones. La ausencia de métodos de investigación con objeto de establecer una relación causal entre diversas variables, de métodos estadísticos y de la temática misma sobre bibliotecas públicas y universitarias, queda probada en análisis temáticos y metodológicos recientes sobre la investigación en biblioteconomía y documentación en las principales revistas científicas de nuestro país (Ferran-Ferrer et al., 2017; Guallar et al., 2017). Sin embargo sí que podemos destacar, por ejemplo, estudios que relacionan el presupuesto de una biblioteca universitaria con la productividad investigadora de la ins-

titución (Fernández; Rubio, 2013). En este sentido existe también lo que se nombra como calculadoras del valor económico de los servicios prestados por las bibliotecas, como por ejemplo la del Consejo Superior de Investigaciones Científicas (CSIC), que analizan el retorno de la inversión relacionando precisamente la inversión en bibliotecas con los beneficios que se obtendrían de su uso (CSIC, s.f.).

Eso mismo nos lleva a dirigir nuestra atención al concepto de eficiencia, por cuanto se hace necesario conocer no sólo si las bibliotecas cumplen sus objetivos (su eficacia) sino también la capacidad que tienen de conseguir las máximas salidas con el mínimo de recursos posibles (su eficiencia). Bustos-González (2007) se pregunta qué sabemos sobre eficiencia en bibliotecas y plantea que el instrumental de indicadores es abundante en medidas de eficacia pero no de eficiencia. También advierte de que si no contamos con un adecuado instrumental para medir los resultados e impactos de las bibliotecas en las universidades difícilmente podemos estar seguros de si aportamos valor a la institución de la que formamos parte, algo que podemos extrapolar a cualquier tipo de biblioteca.

En el estudio de la eficiencia en bibliotecas españolas podemos mencionar la aplicación de la técnica de análisis envolvente de datos (DEA) a las bibliotecas de la Universidad Complutense de Madrid (Simon-Blas; Arias-Coello; Simón-Martín, 2007) y a las bibliotecas universitarias españolas en la gestión de personal (Simón-Martín; Simón-Blas; Arias-Coello, 2016). Este último trabajo es de los pocos mencionados que analiza el conjunto de bibliotecas universitarias españolas ofreciendo un ranking de las mismas. Aunque en ambos trabajos se incluyen variables relacionadas con los recursos (en el primero: suma de personal técnico, auxiliar y de servicio y número de becarios, número de volúmenes y revistas vivas y superficie de la biblioteca; y en el segundo: volumen de fondo informatizado, volúmenes añadidos a la colección y número de puntos de servicio disponibles para los usuarios), en ninguno se incluye el presupuesto. 
En cuanto a bibliotecas públicas, en la sección de indicadores de rendimiento de la página $B i-$ bliotecas públicas españolas en cifras (MECD, 2015) encontramos indicadores denominados de eficiencia. Pero si accedemos a ellos podemos comprobar que sólo atienden a los gastos y no a la relación de los mismos (en virtud de los mínimos) con las salidas (en virtud de las máximas). En un sentido similar la Federación Internacional de Asociaciones de Bibliotecarios y Bibliotecas (IFLA) contempla en su sección de estadísticas anuales para las bibliotecas metropolitanas una serie de rankings que igualmente atienden sólo a una parte de las bibliotecas, como por ejemplo el de la circulación per capita, colección per capita, etc., sin incluir alguno de eficiencia de las bibliotecas relacionando recursos con salidas (IFLA, 2012). Lo mismo sucede con los indicadores propuestos en la perspectiva sobre eficiencia de la norma ISO 11620:2014 refiriéndose al coste total por usuario sin poner en relación ese coste con algún indicador de la perspectiva ISO (ISO, 2014a). La relación entre los costes (entradas) y los usos (salidas) resulta esencial y necesaria en el análisis de la eficiencia.

En base a ello hemos trabajado por superar la carencia en indicadores y medidas sobre eficiencia en bibliotecas elaborando indicadores de segundo nivel gracias a la metodología basada en la teoría general de sistemas. Para ello se han tenido en cuenta los criterios sobre la sencillez y precisión de la formulación y la economía de recursos en los procedimientos de obtención de los datos para diseñar nuevos indicadores de la última norma mencionada (ISO, 2014a). Los indicadores que se han elaborado se basan en los ya conocidos, que podemos calificar de primer nivel, planteados en la anterior norma. En este artículo presentamos los resultados de la aplicación de la metodología empleada como herramienta digital abierta a las bibliotecas universitarias y las redes de lectura pública españolas en forma de rankings.

\section{Secaba-Rank}

\subsection{Objetivos}

Nos marcamos los siguientes:

- Elaborar una metodología para medir la eficiencia de las bibliotecas y conocer mediante nuevos indicadores de segundo nivel cuáles son las que procesan mejor las entradas para la obtención de unas salidas.

- Aplicar dicha metodología a las redes de lectura pública y a las bibliotecas universitarias españolas para conocer las más eficientes encontrando modelos de buenas prácticas para la mejora, especialmente de las que más lo necesitan (benchmarking).

- Convertir Secaba-Rank en un punto de información sobre las redes de lectura pública y las bibliotecas universitarias españolas a la que acudir para los implicados en la toma de decisiones o gestores, e interesados, y punto del que partir en investigaciones futuras sobre el tema.

\subsection{Metodología}

En el estudio se parte de unos datos considerados como mediciones de la realidad, que se procesan para obtener información gracias al cálculo de indicadores. La fuente de datos para Secaba-Rank Universitarias es Rebiun (Rebiun, s.f.) y para Secaba-Rank Públicas la web Bibliotecas públicas españolas en cifras (MECD, 2015). Ambas herramientas incluyen resultados para los años 2007 y los comprendidos entre 2010 y 2015 (incluidos). La metodología que seguimos se basa en una evaluación con base cuantitativa y empírica y desde un enfoque sistémico. Este enfoque que considera a la biblioteca como sistema se explica a partir de la figura 2 .

Siguiendo la figura 2, la biblioteca se considera un sistema con unas entradas (por lo general medibles en euros) que afectan a la entidad (personal, equipamiento, colección, etc.) y alcanzando unas salidas (circulación: préstamos, etc.). Además de las salidas, la biblioteca genera una serie de beneficios (aumento del capital humano, social y cultural...) que son más difíciles de cuantificar (ISO, 2014b). Considerada como sistema, la biblioteca se encuentra en un entorno del cual recibe las entradas como financiación por los beneficios que dicho entorno espera recibir y alcanzar gracias a los servicios que ofrece. Por último, la retroalimentación es el momento para la evaluación y en el que el sistema reflexionaría sobre sí mismo para su mejora.

Desde el enfoque planteado se pretende analizar y evaluar las bibliotecas universitarias y las redes de lectura pública españolas. Conviene resaltar que Secaba-Rank consiste más bien en 2 productos diferentes que comparten el enfoque sistémico de análisis y el mismo directorio raíz en su publicación online:

- El entorno de las redes de lectura pública es el de la sociedad o comunidad en que se hallan las bibliotecas públicas, y los beneficios que se derivan de su uso pueden entenderse desde el objetivo de aumentar el capital social, humano y cultural de las personas (Herrera-Viedma; López-Gijón, 2013).

- El entorno propio y más específico del análisis y evaluación de las bibliotecas universitarias españolas es el de la universidad como institución. Los beneficios que se derivan de su uso se enmarcan en el contexto propio también de la universidad, por ejemplo, como recurso para los estudiantes en sus logros y éxitos académicos, o como vía 
esencial en las tareas de investigación (Bustos-González, 2007). También estos beneficios más específicos conllevarían un aumento de los beneficios para la sociedad en general.

Como comunidades autónomas más potentes destacan la Comunidad Foral de Navarra, Región de Murcia, La Rioja, Castilla y León, y Catalunya, y como más dispotentes, Canarias y Andalucía

A pesar de la concretización de elementos del sistema entre las redes de lectura pública y las bibliotecas universitarias españolas, las bibliotecas de diferente tipo se pueden analizar y evaluar con la metodología desarrollada gracias al enfoque propuesto de la biblioteca como sistema. Argumentando y razonando en base a dicha metodología el análisis concreto de la eficiencia de bibliotecas de diferentes tipos e incluso de distintos servicios bibliotecarios, conviene resaltar que lo importante no serían sólo los elementos del sistema biblioteca, sino también la relación que se da y podría darse entre ellos. Las relaciones planteadas equivaldrían a las flechas en la figura 1 y la atención a las mismas permite el análisis de las relaciones entre los elementos del sistema biblioteca. Desde ahí podrían ser planteadas cuestiones tales como en qué grado elementos como las salidas o los beneficios están configurados por las entradas o la entidad. El hecho de relacionar las entradas con las salidas facilita alcanzar información precisamente del procesamiento que cada entidad y biblioteca realiza de las entradas, conociendo qué bibliotecas son las que mejor las están procesando. Es esto lo que más interesa en relación con la eficiencia en bibliotecas, sea cual sea su tipo, y sobre distintos servicios de las mismas, y lo que facilitan los indicadores de segundo nivel propuestos.

\subsection{Indicadores de segundo nivel}

\section{Potencia del sistema}

Este indicador pone en relación las entradas con las salidas ofreciendo información de cómo son procesadas las primeras por la entidad. Requiere indicadores de primer nivel relacionados con las entradas (presupuesto per capita) y con las salidas (circulación per capita, visitas, actividades culturales, etc.).

Tanto en Secaba-Rank Universitarias como en Secaba-Rank Públicas se ha utilizado en referencia a las entradas el presupuesto per capita para el personal y para adquisiciones. En las salidas, en el primer caso se han incluido los préstamos domiciliarios y los documentos descargados de los recursos electrónicos del apartado "datos Counter", y en el segundo los préstamos totales a usuarios. La población en Secaba-Rank Universitarias la forman la suma de estudiantes de grado, posgrado y títulos propios, y los docentes con dedicación completa y parcial; en el caso de Secaba-Rank Públicas la población de cada comunidad autónoma y/o provincia (según sea el tipo de análisis).

A partir de estos indicadores de primer nivel se calculan la potencia de entrada y la potencia de salida. La potencia de entrada no es más que el resultado de dividir el presupuesto per capita de cada sistema (biblioteca o red) entre el presupuesto per capita del total (que llamamos base), y de igual forma con los préstamos y salidas. Si por ejemplo quisiéramos saber la potencia de entrada de la Comunidad Foral de Navarra, dividiríamos su presupuesto per capita entre el presupuesto per capita del total de España. Con todo lo anterior, la potencia del sistema es el resultado de la división de la potencia de salida entre la de entrada:

Potencia del sistema $=$ Potencia de salida $/$ Potencia de entrada

La potencia del sistema puede ser también despejada desde el principio general de la circulación formulado por López-Gijón (2003) y por el que se ve claramente que las salidas de una biblioteca en tanto que sistema están afectadas y condicionadas por las entradas que se le dan y por el procesamiento de las mismas por la entidad. La formulación de dicho principio es la siguiente:

Potencia de salida $=$ Potencia de entrada $*$ Potencia del sistema

En la tabla 1 se exponen los tres estados en los que puede encontrarse una biblioteca de acuerdo con el indicador de la potencia de los sistemas.

El sistema se encuentra en un estado de:

- potencia: cuando su potencia del sistema es mayor que 1 , esto es, cuando su potencia de salida es mayor que la de entrada (eficiencia);

- dispotencia: cuando la potencia del sistema es menor que 1 , esto es, cuando su potencia de entrada es mayor que la de salida (no eficiencia);

- sin potencia: cuando la potencia del sistema es igual a 1 , esto es, cuando la potencia de entrada es igual que la de salida.

\section{Formulación del sistema}

Este indicador es una formulación del anterior y se nombra expresando que el sistema que estamos estudiando con " $x$ " entradas produce " $\mathrm{y}$ " salidas:

Formulación del sistema $=$ Potencia de entrada $\rightarrow$ Potencia de salida

Tabla 1. Estados posibles de una biblioteca según la potencia del sistema

\begin{tabular}{|c|c|c|c|}
\hline Potencia del sistema & Descripción del sistema & Formulación & Estado \\
\hline$P($ sis $)>1$ & La potencia de salida es mayor que la de entrada & $P($ Sal $)>P(E)$ & Potencia \\
\hline$P($ sis $)=1$ & La potencia de salida es igual a la de entrada & $P($ Sal $)=P(E)$ & Sin potencia \\
\hline $\mathrm{P}($ sis $)<1$ & La potencia de salida es menor que la de entrada & $\mathrm{P}(\mathrm{Sal})<\mathrm{P}(\mathrm{E})$ & Dispotencia \\
\hline
\end{tabular}

$\mathrm{P}($ sis) equivale a potencia del sistema, $\mathrm{P}(\mathrm{E})$ a potencia de entrada y $\mathrm{P}(\mathrm{Sal})$ a potencia de salida. 
El interés de este indicador radica en que ofrece información muy sintética acerca de cómo los sistemas están procesando las entradas al tratar la entidad como si fuese una caja negra. Ordenando los sistemas por su potencia de entrada, para saber cuáles son los que tienen entradas similares, podemos ver claramente qué salidas consiguen unos y otros para establecer los modelos de buenas prácticas entre ellos y facilitar el benchmarking.

Tres de las cinco bibliotecas universitarias con mayor potencia pertenecen a universidades de Catalunya

\section{Esfuerzos en presupuesto y en circulación}

Estos indicadores ponen en relación respectivamente el porcentaje de presupuesto y circulación de cada sistema con el de población por medio de la resta:

Esfuerzo en presupuesto $=$ Porcentaje de presupuesto - Porcentaje de población

Esfuerzo en circulación = Porcentaje de circulación - Porcentaje de población

De esta forma se pueden dar tres situaciones:

- que el resultado sea mayor que 0: situación positiva en cuanto a los esfuerzos que lleva a cabo el sistema para su población, pues el porcentaje en presupuesto o circulación sería mayor que el de población;

- que el resultado sea menor que 0: situación negativa que indica que el sistema no estaría haciendo lo necesario en cuanto al presupuesto y circulación para su población, pues el porcentaje de ésta sería mayor que los dos primeros;

- que el resultado sea igual a 0: situación de normalidad pues los porcentajes serían iguales.

Tanto esta metodología como los indicadores expuestos fueron desarrollados por López-Gijón (2003) en su tesis doctoral de título La red de lectura pública en Andalucía: un análisis sistémico, y presentada en López-Gijón y Vílchez-Pardo (2004). Una síntesis de la fundamentación teórica de la metodología y del proceso, que va desde unos datos hasta los modelos que permite, se puede ver en López-Gijón (1997). Ahora se retoma esta metodología en su aplicación a las bibliotecas universitarias y a las redes de lectura pública españolas. Se puede encontrar más información sobre la metodología e indicadores en la sección "Metodología" de Secaba-Rank.

http://secaba.ugr.es/rank/index.php/metodologia

\section{Resultados}

Tanto Secaba-Rank Universitarias como Secaba-Rank Públicas se componen de las mismas secciones:

- Inicio;

- Secaba-Rank: es el ranking para la potencia de los sistemas;

- Formulación del sistema: incluye los resultados de dicho indicador;

- Indicadores: incluye el presupuesto y circulación per capita y los esfuerzos en presupuesto y circulación;
- Series temporales: resultados para cada biblioteca o red para todos los años comprendidos en el estudio;

- Benchmarking: página en la que se pueden seleccionar las redes y bibliotecas que se desean comparar;

- Estimación y predicción: incluye los resultados y la representación gráfica del coeficiente de correlación lineal entre el presupuesto y la circulación per capita para cada año y caso.

En Secaba-Rank Universitarias se pueden utilizar tres filtros para navegar por los resultados:

- año: 2007 y los comprendidos entre 2010 y 2015 incluidos;

- tramo de población: mayor de 40 mil, entre 40 mil y 20 mil, y menores de 20 mil;

- tipo de universidad: pública, privada y no presenciales.

En Secaba-Rank Públicas se incluyen dos filtros:

- año: los mismos que para el caso anterior;

- tipo de análisis: comunidades autónomas y provincias.

En la tabla 2 se muestran los resultados de la potencia del sistema para las comunidades autónomas en 2015 incluidos en la sección Secaba-Rank dentro de Secaba-Rank Públicas. http://secaba.ugr.es/rank/publicas/index.php/secaba-rank

Las redes (sistemas) que están por encima del 1 consiguen un estado de potencia puesto que su potencia de salida es mayor que la de entrada. Destaca la Comunidad Foral de Navarra como la más potente $(1,46)$ seguida de la Región de Murcia (1,37), La Rioja y Castilla y León $(1,36)$, y Catalunya $(1,32)$.

Los que quedan por debajo del 1 son los sistemas dispotentes y se han de destacar las tres últimas posiciones del ranking: País Vasco $(0,77)$, Andalucía $(0,58)$ y Canarias $(0,50)$.

Tabla 2. Potencia de los sistemas 2015 en Secaba-Rank Públicas (comunidades autónomas)

\begin{tabular}{|c|c|c|c|}
\hline Posición & Red & $\begin{array}{l}\text { Potencia del } \\
\text { sistema }\end{array}$ & Estado \\
\hline 1 & Comunidad Foral de Navarra & 1,46 & Potencia \\
\hline 2 & Región de Murcia & 1,37 & Potencia \\
\hline 3 & La Rioja & 1,36 & Potencia \\
\hline 3 & Castilla y León & 1,36 & Potencia \\
\hline 4 & Catalunya & 1,32 & Potencia \\
\hline 5 & Aragón & 1,17 & Potencia \\
\hline 6 & Principado de Asturias & 1,15 & Potencia \\
\hline- & Total redes & 1,00 & Base \\
\hline 7 & Castilla-La Mancha & 0,96 & Dispotencia \\
\hline 8 & Comunidad Valenciana & 0,95 & Dispotencia \\
\hline 9 & Cantabria & 0,94 & Dispotencia \\
\hline 9 & Illes Balears & 0,94 & Dispotencia \\
\hline 10 & Extremadura & 0,88 & Dispotencia \\
\hline 11 & Comunidad de Madrid & 0,87 & Dispotencia \\
\hline 12 & Galicia & 0,86 & Dispotencia \\
\hline 13 & País Vasco & 0,77 & Dispotencia \\
\hline 14 & Andalucía & 0,58 & Dispotencia \\
\hline 15 & Canarias & 0,50 & Dispotencia \\
\hline
\end{tabular}


Respecto a los casos de Andalucía y Canarias, en el ranking para 2015 cabe resaltar que han perdido incluso en potencia del sistema respecto de 2014 (Andalucía pasó de 0,63 a 0,58 y Canarias de 0,53 a 0,50).

http://secaba.ugr.es/rank/publicas/index.php/secabarank/?wdt_column_filter[Año]=2014

En cambio el País Vasco ha aumentado su potencia del sistema de 0,64 en 2014 a 0,77 en 2015, resultado y consecuencia que puede deberse a la fuerte inversión y el esfuerzo en presupuesto que realiza, siendo la comunidad que más invierte tal y como se observa en la sección de Indicadores (13,04 en presupuesto per capita, y sólo por detrás de Catalunya en esfuerzo en presupuesto, con 3,28), y con una fuerte inversión constante a lo largo de los años como se puede ver en sus series temporales (desde 2010 nunca baja de los $12 €$ per capita y siempre alrededor del doble que para el total de España). http://secaba.ugr.es/rank/publicas/index.php/indicadores http://secaba.ugr.es/rank/publicas/index.php/seriestemporales/?wdt_column_filter[Red] $=($ CCAA) País Vasco

Gracias a nuevos instrumentos y métodos se ha alcanzado una nueva percepción y conocimiento de la realidad de las bibliotecas

El caso concreto del País Vasco en 2015 , en cuanto a potencia del sistema puede ser comprendido por el principio general de circulación expuesto anteriormente. Si las salidas en una biblioteca están condicionadas por las entradas, los sistemas con mayores entradas tenderán a ser los que mayores salidas consigan. También es esencial atender al funcionamiento de la entidad y al procesamiento que lleva a cabo de las entradas, pues las salidas también dependen de ese procesamiento, y eso es algo que recoge a la perfección el indicador de la potencia del sistema. Lo que hay que tener en cuenta es que si la eficiencia depende de los recursos económicos, su estudio y la interpretación de los resultados requiere que seamos conscientes de que la inversión y su aumento precisa de tiempo para poder ser apreciada en la realidad de las bibliotecas y en las investigaciones que se lleven a cabo sobre ello.

En la tabla 3 se muestran los resultados de la potencia del sistema para las bibliotecas universitarias en 2015 incluidos en la sección Secaba-Rank dentro de Secaba-Rank Universitarias. http://secaba.ugr.es/rank/universitarias/index.php/secaba-rank

El análisis en cuanto a estados del sistema es similar al caso de Secaba-Rank Públicas. En este caso se han incluido en la misma tabla las bibliotecas de universidades públicas, privadas y no presenciales. Aunque la mayoría de las privadas son las que ocupan los últimos puestos en el ranking, también las hay que son potentes:

- Universidad Europea de Madrid: potencia del sistema de 1,27 y en la posición 10 ;

- Universitat Internacional de Catalunya: 1,26 y posición 11;

- Universidad de Navarra: 1,19 y posición 13.
En los puestos más altos y por tanto como bibliotecas más eficientes destacan la de la Universitat Rovira i Virgili $(2,08)$, Universitat Politècnica de València $(1,82)$, Universitat Autònoma de Barcelona $(1,79)$, Universidad Autónoma de Madrid $(1,54)$ y Universitat de Barcelona $(1,48)$. Cabe resaltar que tres de estas cinco bibliotecas pertenecen a bibliotecas de universidades de Catalunya.

En la tabla 4 podemos ver una selección de 5 bibliotecas universitarias en 2015 en Secaba-Rank Universitarias para un análisis a modo de ejemplo respecto de la sección y el indicador de la formulación del sistema.

http://secaba.ugr.es/rank/universitarias/index.php/ formulacion-del-sistema

El indicador de la formulación del sistema permite obtener una clasificación de los sistemas por su potencia de entrada. En nuestro ejemplo vemos claramente que las bibliotecas de la Universidad Politécnica de Madrid y la Universitat Politècnica de València tienen similar potencia de entrada $(0,82$ y 0,85 respectivamente), pero mientras que la de la primera tan sólo obtiene 0,60 en la potencia de salida, la de la segunda obtiene 1,54. Por ello UPV se convierte en un modelo de buenas prácticas al procesar las entradas de tal forma que genera 2,5 veces más salidas (de ahí también la diferencia en la potencia del sistema: de 0,73 para la biblioteca de la UPM y de 1,82 para la de la UPV). A su vez podemos pensar que algo negativo está pasando en la entidad de la biblioteca de la Politécnica de Madrid puesto que su entidad está actuando como bloqueo de las entradas y no se están aprovechando como sí sucede en la biblioteca de la Universitat Politècnica de València.

\section{Secaba-Rank se convierte en un punto} al que acudir para personas interesadas, investigadores, y agentes y gestores implicados en la toma de decisiones

Similar análisis se podría hacer con las bibliotecas de la Universidad de Murcia, Universidad de Granada y Universitat de Barcelona. Las tres tienen una potencia de entrada similar $(1,05,1,07$ y 1,08 respectivamente) pero distinta potencia de salida $(0,60,1,14$ y 1,60$)$, resultando que la de la Universidad de Granada sería un modelo de buenas prácticas para la de la Universidad de Murcia, y a su vez la de la Universitat de Barcelona para las otras dos.

En relación con la formulación del sistema hemos centrado la atención en la potencia de entrada, pero también podemos centrarla en la de salida. Las bibliotecas de la Universitat Politècnica de València y la Universitat de Barcelona tienen similar potencia de salida $(1,54$ y 1,60$)$, pero mientras que la de la Universitat de Barcelona la consigue con 1,08 en la de entrada, la de la Universitat Politècnica de València la logra con 0,85 , logrando una posición más alta en el ranking (esta última en la segunda posición mientras que aquella en la quinta). La biblioteca de la Universitat Politècnica de València sería un modelo de buenas prácticas al marcar la posibilidad de un proceso de optimización a la biblioteca de la Universitat de Barcelona. 
Tabla 3. Potencia de los sistemas 2015 en Secaba-Rank Universitarias

\begin{tabular}{|c|c|c|c|}
\hline Posición & Biblioteca & Potencia del sistema & Estado \\
\hline 1 & Universitat Rovira i Virgili (URV) & 2,08 & Potencia \\
\hline 2 & Universitat Politècnica de València (UPV) & 1,82 & Potencia \\
\hline 3 & Universitat Autònoma de Barcelona (UAB) & 1,79 & Potencia \\
\hline 4 & Universidad Autónoma de Madrid (UAM) & 1,54 & Potencia \\
\hline 5 & Universitat de Barcelona (UB) & 1,48 & Potencia \\
\hline 6 & Universidad de Sevilla (US) & 1,47 & Potencia \\
\hline 6 & Universidad de Cádiz (UCA) & 1,47 & Potencia \\
\hline 7 & Universidad Pablo de Olavide (UPO) & 1,46 & Potencia \\
\hline 8 & Universidad Miguel Hernández (UMH) & 1,31 & Potencia \\
\hline 9 & Universitat Pompeu Fabra (UPF) & 1,28 & Potencia \\
\hline 10 & Universidad Europea de Madrid (UEM) (priv.) & 1,27 & Potencia \\
\hline 11 & Universitat Internacional de Catalunya (UIC) (priv.) & 1,26 & Potencia \\
\hline 11 & Universitat de València (UV) & 1,26 & Potencia \\
\hline 12 & Universitat Jaume I (UJ) & 1,23 & Potencia \\
\hline 12 & Universidad de Córdoba (UCO) & 1,23 & Potencia \\
\hline 13 & Universidad de Navarra (UN) (priv.) & 1,19 & Potencia \\
\hline 14 & Universitat Politècnica de Catalunya (UPC) & 1,17 & Potencia \\
\hline 15 & Universidad de Oviedo (UO) & 1,12 & Potencia \\
\hline 16 & Universidad de Málaga (UMA) & 1,10 & Potencia \\
\hline 17 & Universidad de Jaén (UJA) & 1,08 & Potencia \\
\hline 18 & Universidad de Granada (UGR) & 1,07 & Potencia \\
\hline 19 & Universidad del País Vasco (UPV/EHU) & 1,01 & Potencia \\
\hline- & Total Rebiun & 1 & Base \\
\hline 20 & Universidad Católica San Antonio de Murcia (UCAM) (priv.) & 0,92 & Dispotencia \\
\hline 21 & Universidad Europea Miguel de Cervantes (UEMC) (priv.) & 0,89 & Dispotencia \\
\hline 22 & Universitat de Girona (UdG) & 0,88 & Dispotencia \\
\hline 23 & Universidad Carlos III de Madrid (UC3M) & 0,87 & Dispotencia \\
\hline 24 & Universidad de Salamanca (USAL) & 0,82 & Dispotencia \\
\hline 25 & Universidade da Coruña (UDC) & 0,78 & Dispotencia \\
\hline 26 & Universidad Politécnica de Madrid (UPM) & 0,73 & Dispotencia \\
\hline 27 & Universidad de Castilla-La Mancha (UCLM) & 0,69 & Dispotencia \\
\hline 28 & Universidad Complutense (UCM) & 0,68 & Dispotencia \\
\hline 28 & Universidad Nacional de Educación a Distancia (UNED) (no presencial) & 0,68 & Dispotencia \\
\hline 28 & Universidad de Zaragoza (UNIZAR) & 0,68 & Dispotencia \\
\hline 28 & Universitat de Lleida (UdL) & 0,68 & Dispotencia \\
\hline 29 & Universidad de Huelva (UHU) & 0,67 & Dispotencia \\
\hline 30 & Mondragon Unibertsitatea (UMON) (priv.) & 0,62 & Dispotencia \\
\hline 31 & Universidad Pública de Navarra (UPN) & 0,60 & Dispotencia \\
\hline 31 & Universidad de Las Palmas de Gran Canaria (ULPGC) & 0,60 & Dispotencia \\
\hline 31 & Universidad de Cantabria (UC) & 0,60 & Dispotencia \\
\hline 32 & Universidad de La Rioja (UR) & 0,58 & Dispotencia \\
\hline 32 & Universidad de Murcia (UM) & 0,58 & Dispotencia \\
\hline 33 & Universidad de Alcalá de Henares (UAH) & 0,54 & Dispotencia \\
\hline 34 & Universidad de La Laguna (ULL) & 0,53 & Dispotencia \\
\hline 34 & Universidad Alfonso XEI Sabio (UAX) (priv.) & 0,53 & Dispotencia \\
\hline 35 & Universidad de Extremadura (UEX) & 0,52 & Dispotencia \\
\hline 36 & Universitat d'Alacant (UA) & 0,51 & Dispotencia \\
\hline 36 & Universitat Abat Oliba CEU (UAOCEU) (priv.) & 0,51 & Dispotencia \\
\hline 37 & Universidad Rey Juan Carlos (URJC) & 0,49 & Dispotencia \\
\hline 38 & Universidad Pontificia de Salamanca (UPSA) (priv.) & 0,46 & Dispotencia \\
\hline 39 & Universidad de Valladolid (UVA) & 0,44 & Dispotencia \\
\hline 40 & Universidad Loyola Andalucía (ULA) (priv.) & 0,38 & Dispotencia \\
\hline 41 & Universidad Antonio de Nebrija (UANE) (priv.) & 0,35 & Dispotencia \\
\hline 42 & Universidad Internacional de Andalucía (UNIA) & 0,25 & Dispotencia \\
\hline 43 & Universidad Cardenal Herrera-CEU (CEU-UCH) (priv.) & 0,15 & Dispotencia \\
\hline
\end{tabular}


Tabla 4. Formulación del sistema en 5 bibliotecas universitarias 2015

\begin{tabular}{|l|c|c|c|}
\hline Biblioteca & Formulación del sistema & Potencia del sistema & Estado \\
\hline Universidad Politécnica de Madrid (UPM) & $0,82 \rightarrow 0,60$ & 0,73 & Dispotencia \\
\hline Universitat Politècnica de València (UPV) & $0,85 \rightarrow 1,54$ & 1,82 & Potencia \\
\hline Universidad de Murcia (UM) & $1,05 \rightarrow 0,60$ & 0,58 & Dispotencia \\
\hline Universidad de Granada (UGR) & $1,07 \rightarrow 1,14$ & 1,07 & Potencia \\
\hline Universitat de Barcelona (UB) & $1,08 \rightarrow 1,60$ & 1,48 & Potencia \\
\hline
\end{tabular}

Cabe resaltar también que la sección para la formulación del sistema se complementa con la de Benchmarking al poder obtener las tablas y gráficos para los distintos indicadores seleccionando las bibliotecas que se deseen.

http://secaba.ugr.es/rank/universitarias/index.php/ benchmarking

Un procedimiento recomendable es acudir a la sección de formulación del sistema para ver qué bibliotecas pueden actuar de modelo de buenas prácticas para otras y después ir a la de Benchmarking seleccionándolas para completar el análisis.

Por último y en cuanto a la sección de Estimación y predicción queremos añadir que consiste en el cálculo del coeficiente de correlación lineal entre el presupuesto y la circulación per capita, y su representación gráfica. En cada caso se ha estudiado la normalidad de las variables calculando bien el coeficiente de Pearson, bien el de Spearman. En la herramienta hemos utilizado Highcharts cloud para la representación gráfica de los resultados en muchas de las secciones ofreciendo gráficos dinámicos (por los que nos podemos mover, hacer zoom, obtener una leyenda para cada objeto representado y decidir qué variables son las que queremos ver).

https://cloud.highcharts.com

En el gráfico 1 se presenta a modo de ejemplo el gráfico de dispersión para el coeficiente de correlación lineal de 2015 en Secaba-Rank Públicas por comunidades autónomas disponible en la sección de Estimación y predicción.

http://secaba.ugr.es/rank/publicas/ index.php/estimacion-y-prediccion

El cálculo del coeficiente de Pearson resulta de 0,78 y por ello la correlación es positiva y cercana a ser muy alta. Gracias a la línea de tendencia vemos los sistemas que obtienen una situación mejor de la esperada (dibujados en verde) y los que no obtienen la circulación estimada de acuerdo al modelo (en rojo). Los sistemas por encima y por debajo de lo esperado son justamente los sistemas potentes y dispotentes según el indicador de la potencia del sistema. La importancia de esta representación gráfica es doble:

- es una herramienta para la predicción y podría ser considerada un mapa de la lectura pública en España al dejar clara la diferencia autónomas 2015 entre la situación real y esperada respecto de las entradas y salidas de los sistemas en un ejercicio de evaluación en aras a su mejora.

- permite comprobar que las salidas de los sistemas están condicionadas por las entradas que se les suministran.

La herramienta Secaba-Rank se convierte en un punto al que acudir para obtener información sobre bibliotecas y, más importante aún, un punto del que partir en futuras investigaciones. Los resultados comentados son tan sólo una muestra concreta en torno a los indicadores de la potencia y la formulación del sistema. Aunque en la elaboración de este artículo se haya tenido que limitar la exposición de resultados, como muestra de la potencialidad de la herramienta sí que se pueden plantear interrogantes para futuras investigaciones en torno a los dos indicadores anteriores y el resto de los contemplados en la herramienta. Por ejemplo:

- ¿Cuáles son los modelos de buenas prácticas para una biblioteca universitaria o red de lectura pública concreta?

- ¿Qué sucede con las bibliotecas universitarias que conforman consorcios?

- ¿Son estas bibliotecas las que más salidas obtienen por compartir recursos y las más eficientes?

- ¿Existe relación entre factores del entorno socioeconómico como pueden ser la renta per capita, el nivel de educación o la tasa de desempleo, y los préstamos y eficiencia de las redes de lectura pública?

- etc.

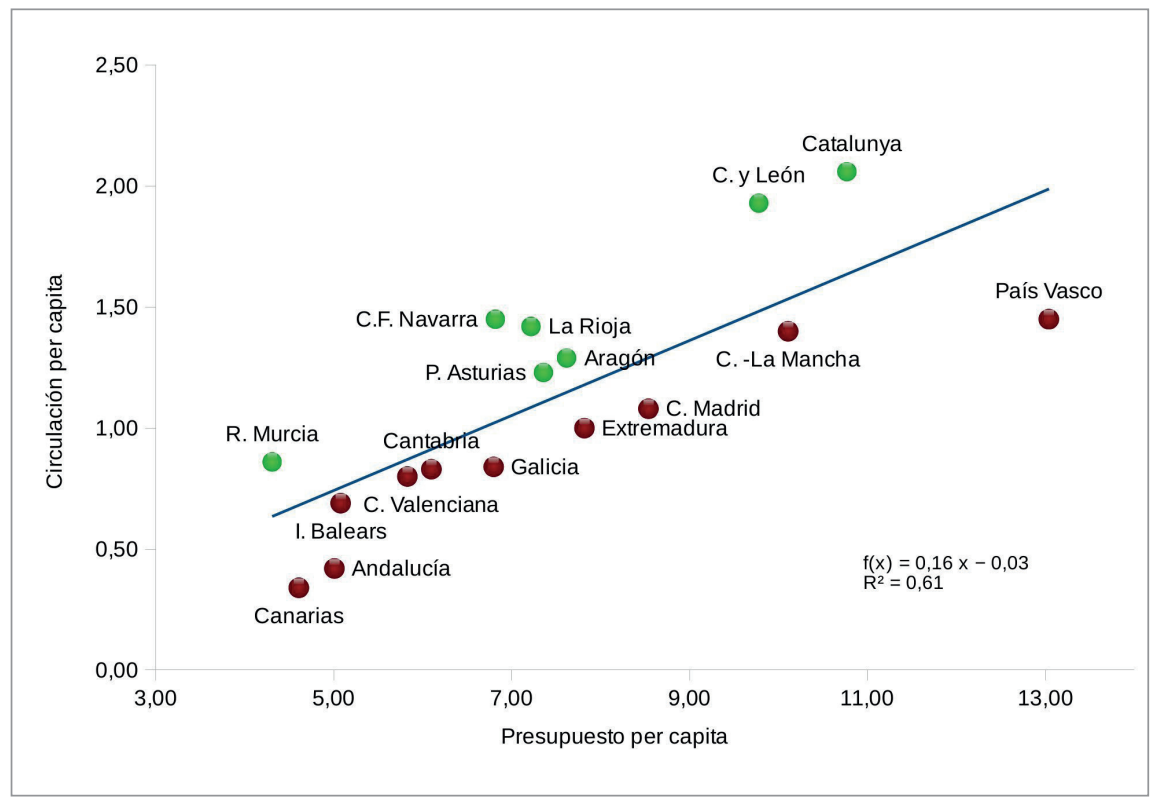

Gráfico 1. Correlación entre el presupuesto per capita y la circulación per capita en comunidades 


\section{Discusión y conclusiones}

Tanto en la elaboración de la metodología como en la implementación de Secaba-Rank como herramienta digital abierta nos marcamos el precepto y el objetivo de que gracias a nuevos instrumentos y métodos seríamos capaces de alcanzar una nueva percepción y conocimiento de la realidad de las bibliotecas. Este nuevo conocimiento como información original y valiosa es lo que hemos alcanzado para la retroalimentación y mejora de las bibliotecas concretamente en la aplicación de la metodología a las bibliotecas universitarias y las redes de lectura pública españolas. Todo ello supone que desde flujos de datos y mediciones de la realidad de los que partimos en la investigación se puedan obtener flujos de control para dirigir a las bibliotecas a alcanzar sus objetivos tanto en el sentido de la eficacia como en el de la eficiencia.

Para ello es importante la fiabilidad de los datos al ser la base de toda investigación. En ese sentido es digna de mencionar la labor que se hace desde Rebiun y el MECD en la recopilación de datos sobre bibliotecas universitarias y públicas, aunque se pueda plantear en futuros trabajos la necesidad de renovación y mejora de los formularios de toma de esos datos y su fiabilidad. Lo que sí es cierto es que gracias a ellos hemos podido llevar a cabo nuestro estudio, permitiendo que Secaba-Rank se convierta así en un punto al que acudir para obtener información sobre bibliotecas y lectura pública para personas interesadas y agentes y gestores en la toma de decisiones al respecto. Además Secaba-Rank se convierte en un punto del que partir en futuras investigaciones sobre bibliotecas y lectura pública.

Por último y en cuanto a perspectivas de futuro, los indicadores de segundo nivel desarrollados para medir la eficiencia contemplan variables concretas tanto en entradas (presupuesto para el personal y en adquisiciones) como en salidas (préstamos y documentos descargados). Pero la biblioteca se caracteriza no sólo por ser un servicio relacionado con el préstamo de volúmenes, sino que se ha convertido en un servicio esencial para la comunidad (Herrera-Viedma; López-Gijón, 2013). Nuestro objetivo para el futuro es trabajar por incluir nuevas variables en las salidas (por ejemplo: visitas, actividades culturales, uso de internet, o formación de usuarios) y trabajar en el estudio de la eficiencia referida a la relación entre entradas y beneficios (mayor capital cultural, social y humano) de las bibliotecas y no sólo entre entradas y salidas.

\section{Agradecimientos}

Pedro Lázaro-Rodríguez es beneficiario de una de las ayudas para contratos predoctorales de Formación de Profesorado Universitario del Ministerio de Educación, Cultura y Deporte (Referencia FPU-2014/04213). El trabajo está soportado por el Ministerio de Economía y Competitividad (Referencia TIN2016-75850-R).

\section{Referencias}

Bustos-González, Atilio (2007). "Bibliotecas universitarias: ¿sabemos medir sus resultados e impactos?". El profesional de la información, julio-agosto, v. 16, n. 4, pp. 281-286. https://doi.org/10.3145/epi.2007.jul.01
Cabrerizo, Francisco-Javier; López-Gijón, Javier; Martínez-Sánchez, María-Ángeles; Morente-Molinera, Juan-Antonio; Herrera-Viedma, Enrique (2017). "A fuzzy linguistic extended LibQUAL+ model to assess service quality in academic libraries". International journal of information technology \& decision making, v. 16, n. 1, pp. 225-244. https://doi.org/10.1142/S0219622015500406

Cabrerizo, Francisco-Javier; Morente-Molinera, Juan-Antonio; Pérez, Ignacio-Javier; López-Gijón, Javier; Herrera-Viedma, Enrique (2015). "A decision support system to develop a quality management in academic digital libraries". Information sciences, v. 323, pp. 48-58.

https://goo.gl/DPXA9v

https://doi.org/10.1016/j.ins.2015.06.022

Consorci de Biblioteques de Barcelona (s.f.). Projecte Tibidabo: Estudi comparatiu de la situació i l'evolució de Xarxes de Biblioteca Pública. Consorci de Biblioteques de Barcelona; Servei de Biblioteques de la Diputació de Barcelona. http://tibidaboproject.bbcn.cat

CSIC (s.f.). Calculador del valor económico de los servicios prestados por la biblioteca. Consejo Superior de Investigaciones Científicas.

http://bibliotecas.csic.es/calculador

Fernández, Sergio; Rubio, Francisco (2013). “¿El dinero importa? Relación entre el presupuesto de la biblioteca y la productividad investigadora de la Universitat Politècnica de València". Revista española de documentación científica, v. 36, n. 4, e023.

https://doi.org/10.3989/redc.2013.4.1043

Ferran-Ferrer, Núria; Guallar, Javier; Abadal, Ernest; Server, Adán (2017). "Research methods and techniques in Spanish library and information science journals (2012-2014)". Information research, v. 22, n. 1, paper 741. http://InformationR.net/ir/22-1/paper741.htm/

Fushimi, Marcela (2011). Evaluación de bibliotecas universitarias: Una propuesta desde las perspectivas objetiva y subjetiva. Buenos Aires: Alfagrama, ISBN: 9789871305629 http://www.memoria.fahce.unlp.edu.ar/libros/pm.19/ pm.19.pdf

Gimeno-Perelló, Javier (2008). Evaluación de la calidad en bibliotecas: compromiso con lo público. Buenos Aires: Alfagrama, ISBN: 9789871305452

González-Fernández-Villavicencio, Nieves; Cánovas-Álvarez, Encarnación; Arahal-Junco, Consuelo (2014). "Evaluación del servicio de referencia de una biblioteca universitaria: Biblioteca de la Universidad de Sevilla". Revista española de documentación científica, v. 37, n. 2, e045.

https://doi.org/10.3989/redc.2014.2.1072

Guallar, Javier; Ferran-Ferrer, Núria; Abadal, Ernest; Server, Adán (2017). "Revistas científicas españolas de información y documentación: análisis temático y metodológico". El profesional de la información, v. 26, n. 5, pp. 947-960. https://doi.org/10.3145/epi.2017.sep.16

Hernández-Sánchez, Hilario; Arroyo-Vázquez, Natalia (2014). "Efectos de la crisis económica en las bibliotecas españolas". El profesional de la información, v. 23, n. 2, pp. 158-164. 
https://doi.org/10.3145/epi.2014.mar.08

Herrera-Viedma, Enrique; López-Gijón, Javier (2013). “Libraries' social role in the information age". Science, v. 339, n. 6126, p. 1382.

https://doi.org/10.1126/science.339.6126.1382-a

IFLA (2012). Annual statistical survey. International Federation of Library Associations and Institutions.

http://www.ifla.org/node/8106

ISO (2014a). ISO 11620:2014. Information and documentation. Library performance indicators.

https://www.iso.org/standard/56755.htm/

ISO (2014b). ISO 16439:2014. Information and documentation. Methods and procedures for assessing the impact of libraries.

https://www.iso.org/standard/56756.html

López-Gijón, Javier (1997). "Desde los datos, hacia los modelos". Educación y biblioteca, n. 78, pp. 44-49.

http://eprints.rclis.org/9079/1/Datos_modelos.pdf

López-Gijón, Javier (2003). “La red de lectura pública de Andalucía: un análisis sistémico". Tesis doctoral. Granada: Universidad de Granada.

http://digibug.ugr.es/bitstream/10481/29141/1/LopezGijonJavier.pdf

López-Gijón, Javier; Ávila-Fernández, Belén; Pérez-Gálvez, Ignacio-Javier; Herrera-Viedma, Enrique (2010). "La calidad en las bibliotecas universitarias biomédicas según sus usuarios". El profesional de la información, v. 19, n. 3, pp. 255-259. https://doi.org/10.3145/epi.2010.may.05

López-Gijón, Javier; Vílchez-Pardo, Josefina (2004). “La titulación de biblioteconomía y documentación y las bibliotecas andaluzas: que veinte años no son nada". Boletín de la Asociación Andaluza de Bibliotecarios, n. 75-76, pp. 193-226. http://eprints.rclis.org/6777/1/75a17.pdf

Martínez-Cardama, Sara; Caridad-Sebastián, Mercedes (2016). "Bibliotecas universitarias y cultura digital: indicadores y validación a partir del ranking de Shanghai". Opción, v. 32 , n. 8, pp. $138-155$

MECD (2015). Bibliotecas públicas españolas en cifras. Ministerio de Educación, Cultura y Deporte.

http://www.mecd.gob.es/cultura-mecd/areas-cultura/ bibliotecas/mc/ebp/portada.html

Motos, Sonia (2002). "PAB Redes: un programa de la Fundación Bertelsmann para dinamizar las redes de bibliotecas públicas". El profesional de la información, v. 11, n. 1, pp. 74-77.

http://www.elprofesionaldelainformacion.com/contenidos/2002/ enero/14.pdf

Muñoz-Egido, Daniel; Vianello-Osti, Marina (2017). “Evaluación de usabilidad de los portales web de las bibliotecas universitarias españolas a partir de un modelo heurístico cognitivo-emocional". Revista española de documentación científica, v. 40, n. 1, e165.

https://doi.org/10.3989/redc.2017.1.1379

Rebiun (s.f.). Estadísticas Rebiun. Red de Bibliotecas Universitarias Españolas.

http://rebiun.um.es

Reques-Velasco, Pedro (2006). Atlas digital de la España universitaria: bases para la planificación estratégica de la enseñanza superior. Santander: Universidad de Cantabria. ISBN: 9788481024142

Rodríguez-Bravo, Blanca; Rodríguez-Sedano, Francisco (2016). "Trends in library collection circulation in Spanish universities: The case of the University of León". Library resources \& technical services, v. 60, n. 4, pp. 248-258.

https://doi.org/10.5860/Irts.60n4.248

Simón-Blas, Clara; Arias-Coello, Alicia; Simón-Martín, José (2007). "Aplicación de la técnica DEA en la medición de la eficiencia de las bibliotecas de la Universidad Complutense de Madrid". Revista española de documentación científica, v. 30, n. 1, pp. 9-23.

https://doi.org/10.3989/redc.2007.v30.i1.366

Simón-Martín, José; Arias-Coello, Alicia; Simón-Blas, Clara (2016). "The impact of the economic crisis on Spanish university libraries". Revista española de documentación científica, v. 39, n. 3, e142.

https://doi.org/10.3989/redc.2016.3.1346

Simón-Martín, José; Simón-Blas, Clara; Arias-Coello, Alicia (2016). "Ranking de las bibliotecas universitarias españolas en la gestión del personal". Revista española de documentación científica, v. 39, n. 1, e119.

https://doi.org/10.3989/redc.2016.1.1259

Taladriz-Mas, Margarita (2013). "Los servicios de información y el retorno de la inversión: cómo llegar a conocerlo". El profesional de la información, v. 22, n. 4, pp. 281-285. https://doi.org/10.3145/epi.2013.jul.01

Von-Bertalanffy, Ludwig (1969). General system theory: Foundations, development, applications. New York: Braziller

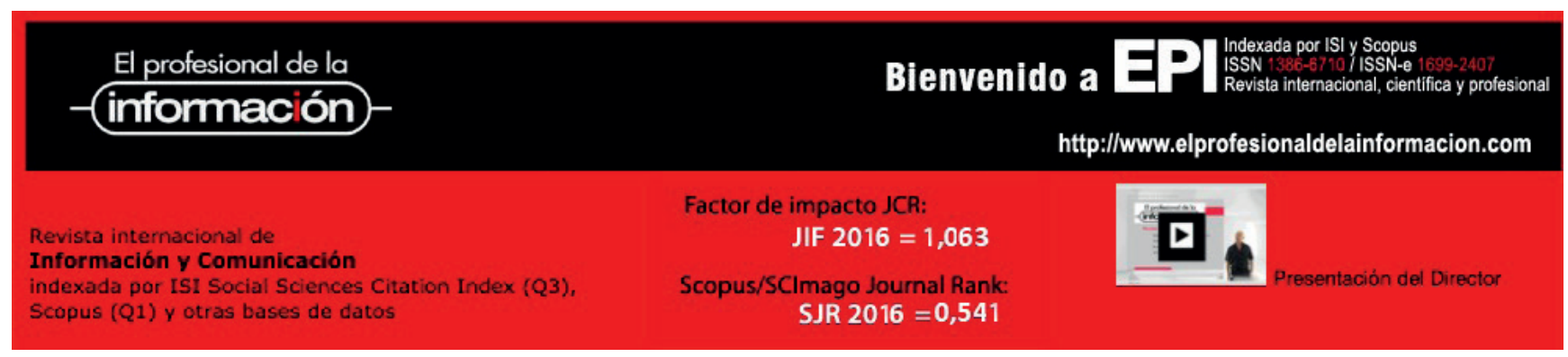

